\title{
Efficient parallel computations of flows of arbitrary fluids for all regimes of Reynolds, Mach and Grashof numbers
}

Efficient parallel computations of fluid flows

\author{
M. Mulas
}

Department of Computational Methods for Engineering, CRS4, Center for Research, Development and Advanced Studies in Sardinia, Area of Computational Fluid Dynamics, Uta (Ca), Italy

\section{S. Chibbaro}

Department of Computational Methods for Engineering, CRS4, Center for Research, Development and Advanced Studies in Sardinia, Area of Computational Fluid Dynamics, Uta (Ca), Italy;

Dipartimento di Fisica, Universita di Cagliari, and INFN, sezione di Cagliari, Cittadella Universitaria di Monserrato, Monserrato (Ca), Italy

\section{G. Delussu, I. Di Piazza and M. Talice}

Department of Computational Methods for Engineering, CRS4, Center for Research, Development and Advanced Studies in Sardinia, Area of Computational Fluid Dynamics, Uta (Ca), Italy

Keywords Computations, Flow, Fluid

Abstract This paper presents a unified numerical method able to address a wide class of fluid flow problems of engineering interest. Arbitrary fluids are treated specifying totally arbitrary equations of state, either in analytical form or through look-up tables. The most general system of the unsteady Navier-Stokes equations is integrated with a coupled implicit preconditioned method. The method can stand infinite CFL number and shows the efficiency of a quasi-Newton method independent of the multi-block partitioning on parallel machines. Computed test cases ranging from inviscid hydrodynamics, to natural convection loops of liquid metals, and to supersonic gasdynamics, show a solution efficiency independent of the class of fluid flow problem.

\section{Introduction}

Flows of engineering interest range from high speed aerodynamics to incompressible hydrodynamics, and include low speed reactive flows and heat exchange loops in natural and forced convection, just to name few examples. On the other hand, fluid dynamics applications to different fluids bring into the mathematical formulation different equations of state, which in turn determine

International Journal of Numerical Methods for Heat \& Fluid Flow, Vol. 12 No. 6, 2002, pp. 637-657. (C) MCB UP Limited, 0961-5539 DOI 10.1108/0961553021043833 
$\mathrm{HFF}$

12,6

638

different levels of interaction between fluid dynamics and thermodynamics. The above range of thermo-fluid dynamics situations is described, in terms of nondimensional parameters, by Reynolds, Mach, Grashof and Prandtl numbers. If chemical equilibrium also represents a reasonable assumption, then reactive flows are simply treated by defining their suitable state equations where heats of reaction are hidden in the mixture's enthalpies and specific heats.

The desire of computational fluid dynamics (CFD) has long been the possibility to use a unique mathematical formulation together with a unique numerical method to examine the variety of fluid dynamics problems, all of them governed by the Navier-Stokes system of equations. Going back to the infancy of CFD (the 1960s and 1970s), numerical simulations for compressible and incompressible flows represented two classes of methods which only shared the name of the governing equations. After the extraordinary pace of development of CFD methods, during the 1980s and 1990s, in more recent years, many efforts have been made to develop unified numerical approaches to the solution of a wider class of fluid flow problems. For this purpose, either typical "incompressible" methods are generalized for high speed compressible flows, or vice versa, the efficient use of "compressible" algorithms is extended to low speed flows of incompressible fluids by means of suitable preconditioners. The two methods are now more correctly called coupled and segregated solution algorithms.

When the coupled methods are used for incompressible flow situations, the main task is to remove or tackle the singularity of the Navier-Stokes equations when Mach number $M$ approaches zero $(M \rightarrow 0)$. The inviscid matrix of the coupled system of equations becomes in fact ill conditioned and though Mother Nature can cope with it, numerical methods break down. As a result, straightforward use of coupled methods gives severe convergence problems or even breakdown in the presence of regions with very low Mach number.

On the other hand, the incompressible Navier-Stokes approximation cannot be used whenever the fluid compressibility and the flow conditions indicate that kinetic and internal energies can exchange reversible $p d V$ work which is given by the capability of temperature and pressure changes to determine density changes. Somehow, arbitrary values of the nondimensional parameters $\beta \Delta T$ (where $\beta$ represents the fluid compressibility coefficient at constant pressure) and the Mach number $M$, respectively, set the trade off between abandoning the incompressible formulation and paying a price in terms of accuracy of the results.

Plenty of studies can be found in literature focused on the attempt to generalize the numerical methods to a wider class of problems. For low speed flows with variable density, methods based on asymptotic expansion have been developed for example in Guerra and Gustafsson (1986). These methods remove acoustic modes in the limit of $M \rightarrow 0$, avoiding the singularity of the compressible equations. Another family of methods has been proposed by 
Patnaik et al. (1987). In these approaches a pressure correction equation is derived from the energy equation, while density is advanced in time via a continuity equation. The method can compute aeroacoustics and nonstationary low Mach number flows.

Segregated methods can be extended to the compressible case. This approach gives the best perspective in the limit $M \rightarrow 0$, where a well-known incompressible scheme can be recovered. Moreover, difficulties are encountered when tackling the acoustic transonic singularity, which determines a nonconvergence behaviour in the regime $M=\mathfrak{D}(1)$ (see Bijl and Wesseling, 1996). Only the use of a first order upwind scheme allows the algorithm to converge (see Bijl and Wesseling, 1998). Staggered schemes are generally used with these methods to avoid pressure oscillations in the low Mach number region. An exception is the work by Demirdzic et al. (1993), where this approach has been adopted with a nonstaggered scheme. More information can be found in Bijl and Wesseling (2000) and Wesseling et al. (2000).

On the other side of the scientific community, coupled algorithms have been equipped with preconditioning methods in order to cope with eigenvalue spreading. This is done by multiplying the system matrix by a preconditioning matrix which alters the speed of the acoustic waves. This makes their speed the same order of magnitude as the speed of the entropy and shear waves, i.e. the fluid local velocity. In this way a well-conditioned system is recovered, together with good convergence properties.

The pioneering work of Chorin (1967) introduced the method of artificial compressibility, and has to be considered as the oldest contribution to the field. Starting from Chorin's method, Turkel $(1984,1987)$ developed a two-parameter preconditioning matrix, where benefits were limited to the low Mach number region. During the 1980s the work on preconditioning techniques was continued by Peyret and Viviand (1985), while Merkle and co-workers (Choi and Merkle, 1985), initially focused on the Euler equations, and later extended this to include viscous effects and turbulence (Merkle, 1998b; Choi and Merkle, 1993). The procedures followed by these authors are based on preconditioning matrices obtained by asymptotic expansions of the compressible equations. Perturbation expansions for low speed effects, viscous effects and unsteady motions are developed to ensure that the pseudo-time terms are in correct balance with the main physical terms of the equations. The preconditioning matrix of this family can also be postulated simply providing a modified expression for derivatives of the density with respect to the pressure. This preconditioning matrix fashion was first announced by Venkateswaran et al. (1992) for the special case of an ideal gas, and then generalized to an arbitrary equation of state by Weiss et al. (1995, 1997), and by Merkle et al. (1998).

Alternative procedures for developing preconditioning matrices have been pursued by van Leer and co-workers (Lee, 1996; Lee and van Leer, 1993; van Leer et al.; 1991). In general, these methods make use of special variables that
Efficient parallel computations of fluid flows

639 
HFF

12,6

640

symmetrize and simplify the system matrix. The preconditioned matrix is designed in order to obtain a condition number equal to $c_{\mathrm{n}}=$ $1 / \sqrt{1-\min \left(M^{2}, M^{-2}\right)}$, and thus $c_{\mathrm{n}}=1$ in the limit $M \rightarrow 0$ (van Leer, 1991). In Lee (1996) an asymmetric preconditioning matrix is derived to remove the preconditioning sensitivity to the flow angle due to the eigenvector structure. The major weakness of this approach is that extension from ideal gas to a general equation of state is not straightforward.

The approach followed in the present work is in line with the derivation of Weiss and Merkle (Weiss and Smith, 1995; Weiss et al. 1997; Merkle and Venkateswaran, 1998) for the preconditioning matrix structure. The main reason for this choice is that arbitrary fluids, described by arbitrary equations of state in analytical or tabular form, can be naturally treated.

\section{Preconditioned governing system of equations}

The full coupled system of the unsteady Navier-Stokes equations represents the governing equations of all fluid flows. Written in conservative formulation, it is given by:

$$
\begin{gathered}
\frac{\partial \rho}{\partial t}+\frac{\partial}{\partial x_{j}}\left(\rho u_{j}\right)=0 \\
\frac{\partial \rho u_{i}}{\partial t}+\frac{\partial}{\partial x_{j}}\left(\rho u_{i} u_{j}+\delta_{i j} p-\tau_{i j}\right)=\rho g_{i} \\
\frac{\partial \rho E}{\partial t}+\frac{\partial}{\partial x_{j}}\left(\rho H u_{j}-u_{i} \tau_{i j}+q_{j}\right)=\rho \dot{Q}
\end{gathered}
$$

where $g_{i}$ represent the components of the acceleration of gravity, $\dot{Q}$ a volumetric external power source, and where the total energy $E$ includes internal and kinetic energy as well as the gravitational potential $\Phi=g z$. Constitutive relations given by Newton's and Fourier's laws of viscosity and heat conductivity, together with two equations of state, close the mathematical model:

$$
\begin{gathered}
\tau_{i j}=\mu\left(\frac{\partial u_{i}}{\partial x_{j}}+\frac{\partial u_{j}}{\partial x_{i}}\right)-\frac{2}{3} \mu \delta_{i j} \frac{\partial u_{k}}{\partial x_{k}} \\
q_{j}=-\kappa \frac{\partial T}{\partial x_{j}} \\
\rho=\rho(p, T) \quad h=h(p, T)
\end{gathered}
$$

In the framework of equations (1)-(4) compressibilty effects come into play whenever the conditions occur. If density changes are related to temperature 
changes, both buoyancy and the hydrostatic pressure gradient are allowed to play their role; on the other side, high fluid speeds, compared to the speed of sound, generate pressure changes which in turn determine density changes. This picture focuses more on the terms of the equations, than on standard fluid classifications: ideal gasdynamic and liquid metal buoyant flows, for instance, share the same thermodynamic $p d V$ work and the reversible exchange between internal and kinetic energy (namely the compressibility) as the key feature of the fluid flow.

Preconditiong the system (1) is achieved multiplying the unsteady terms by a suitable matrix $\mathbf{P}^{-1}$ or, in an equivalent way, the fluxes and the source terms by its inverse $\mathbf{P}$. System (1) can be written in compact vector form displaying the inviscid and viscous flux vectors components $\vec{F}$ and $\vec{G}$ and the source term array $S$ :

$$
\frac{\partial Q}{\partial t}+\mathbf{P}\left(\frac{\partial F_{x}}{\partial x}+\frac{\partial F_{y}}{\partial y}+\frac{\partial F_{z}}{\partial z}\right)+\mathbf{P}\left(\frac{\partial G_{x}}{\partial x}+\frac{\partial G_{y}}{\partial y}+\frac{\partial G_{z}}{\partial z}\right)=\mathbf{P} S
$$

where the preconditioning matrix is given by $\mathbf{P}=\mathbf{M M}_{\mathrm{m}}^{-1}$. $\mathbf{M}$ represents the Jacobian matrix of the vector of conservative variables $Q(\rho, \rho V, \rho E)$ with respect to the vector of the viscous-primitive variables $Q_{\mathrm{v}}(p, \vec{V}, T) . \mathbf{M}_{\mathrm{m}}$ represents a modified version of $\mathbf{M}$. No modification brings back the original non-preconditioned system $(\mathbf{P}=\mathbf{I})$. Matrices $\mathbf{M}$ and $\mathbf{M}^{-1}$ are given in the Appendix.

The matrix $\mathbf{M}$ (equation (28)) contains arbitrary thermodynamics in terms of derivatives of density and enthalpy with respect to pressure and temperature $\left(\rho_{p}, \rho_{T}, h_{p}, h_{T}\right)$, while the matrix $\mathbf{M}_{\mathrm{m}}$ contains "modified" thermodynamics in terms of $\rho_{p}^{\mathrm{m}}$. To keep the condition number of $\mathfrak{Q}(1)$, rescaling of the characteristic speeds is obtained with the following choice of $\rho_{p}^{\mathrm{m}}$ :

$$
\rho_{p}^{\mathrm{m}}=\frac{1}{V_{p}^{2}}-\frac{\rho_{T}}{\rho h_{T}}
$$

where $V_{p}$, a local preconditioning velocity, plays a crucial role as it should be as low as possible, but not smaller than any local transport velocity for stability considerations:

$$
V_{p}=\min \left[\max \left(u, \frac{v}{\Delta_{x}}, \frac{\alpha}{\Delta_{x}}, \sqrt{\frac{\Delta p}{\rho}}\right), c\right]
$$

Here $\alpha$ represents the thermal diffusivity. The criterion based on the local fluid speed $u$ is dominant in turbulent flows, at high Reynolds numbers. The velocity based on the local pressure gradient prevents vanishing $V_{p}$ at stagnation regions. The two criteria based on diffusion velocities depend strongly on the grid stretching inside the boundary layers, where the flow is viscous or heat
Efficient parallel computations of fluid flows

\section{1}


HFF

12,6

642 conduction dominates. Where the velocity $V_{p}$ based on the maximum characteristic speed grows higher than the local speed of sound, then $V_{p}=c$ and the preconditioning is locally and automatically switched off where the flow is supersonic $\left(\rho_{p}^{\mathrm{m}}=\gamma / c^{2}=\rho_{p}\right.$ and $\left.\mathbf{M}_{\mathrm{m}}=\mathbf{M}\right)$. This is particularly useful in all test cases where stagnation and supersonic regions are both present.

\section{Arbitrary fluid}

In the above described mathematical model, the choice of the working fluid is totally arbitrary. Any chosen fluid can be defined by two equations of state of the form of equation (4) and via the matrix $\mathbf{M}$, which requires the derivatives of density and enthalpy with respect to pressure and temperature. For any pure substance, the required derivatives are given by:

$$
\begin{gathered}
\rho_{p}=\rho_{p}(p, T)=\rho \xi \\
\rho_{T}=\rho_{T}(p, T)=-\rho \beta \\
h_{T}=h_{T}(p, T)=c_{p} \\
h_{p}=h_{p}(p, T)=\frac{1-\beta T}{\rho}
\end{gathered}
$$

where the two compressibility coefficients $\beta$ and $\xi$, respectively, at constant pressure and at constant temperature, have a straightforward analytical definition for both ideal gases and liquids. Strict incompressibility conditions can be obtained providing $\beta \equiv \xi \equiv 0$ as input data.

Moreover, density, enthalpy and their derivatives can be given through lookup tables for an arbitrary set of values of pressure and temperature. This option is useful for treating, for instance, reactive mixtures of gases in chemical equilibrium, where the necessary data can be obtained by a number of chemical equilibrium codes available to the scientific community.

\section{Numerical scheme}

Equations are integrated with a cell-centred finite-volume method on blockstructured meshes. Convective inviscid fluxes are computed by a second order Roe's scheme (Roe, 1981; Hirsch, 1990), based on the decomposition of the Euler equations in waves, so that proper upwinding can be applied to each wave depending on the sign of the corresponding wave speed. This implies an eigenvector decomposition of the matrix of the Euler system. The first order Roe's scheme numerical flux vector is given by: 


$$
F_{i+1 / 2}^{*}=\frac{F_{i}+F_{i+1}}{2}-\frac{1}{2}\left[\mathbf{P}^{-1} \mathbf{R}_{p}\left|\Lambda_{p}\right| \mathbf{L}_{p}\right]_{i+1 / 2}\left(Q_{i+1}-Q_{i}\right)
$$

made of a central part and a matrix dissipation part computed at the cell Efficient parallel computations of fluid flows interface, denoted by $(i+1 / 2)$. $\mathbf{R}_{p}$ and $\mathbf{L}_{p}$ represent the matrices of right and left eigenvectors of:

$$
\mathbf{D}_{p}=\mathbf{P D}=\mathbf{P}\left(\frac{\partial F_{x}}{\partial Q} n_{x}+\frac{\partial F_{y}}{\partial Q} n_{y}+\frac{\partial F_{z}}{\partial Q} n_{z}\right)
$$

and $\left|\Lambda_{p}\right|$ represents the diagonal matrix whose elements $\alpha_{p}$ are the absolute values $\left|\lambda_{p}\right|$ of the eigenvalues of the system matrix $\mathbf{D}_{p}$. Second order accuracy is achieved, (Hirsch, 1990) for a thorough review, computing the elements $\alpha_{p}$ as:

$$
\alpha_{p}=\left|\lambda_{p}\right|+\Psi\left(R^{+}\right)-\Psi\left(R^{-}\right)
$$

with:

$$
R^{+}=\frac{\lambda^{+}\left(Q_{i}-Q_{i-1}\right)}{\lambda^{+}\left(Q_{i+1}-Q_{i}\right)} \quad R^{-}=\frac{\lambda^{-}\left(Q_{i+2}-Q_{i+1}\right)}{\lambda^{-}\left(Q_{i+1}-Q_{i}\right)}
$$

where the function $\Psi$ represents an appropriate limiter which assures monotonicity of the solution at discontinuities and at local minima and maxima. Setting $\Psi=0$ in Equation (11), the first order Roe's scheme is recovered.

The expression of the eigenvectors in terms of conservative variables is rather complicated. Easier algebra and programming can be achieved transforming equation (9) in the equivalent expression for the primitive variables $Q_{\mathrm{v}}$ :

$$
F_{i+1 / 2}^{*}=\frac{F_{i}+F_{i+1}}{2}-\frac{1}{2}\left[\mathbf{M}_{\mathrm{m}} \mathbf{R}_{p}^{\mathrm{v}}\left|\Lambda_{p}\right| \mathbf{L}_{p}^{\mathrm{v}}\right]_{i+1 / 2}\left(Q_{i+1}^{\mathrm{v}}-Q_{i}^{\mathrm{v}}\right)
$$

Euler matrices and eigenvectors in conservative and primitive variables are related by similarity transformations. Preconditioned eigenvectors are given in Appendix A for a general equation of state. Viscous fluxes and source terms are calculated with standard cell-centred finite-volume techniques and they are both second order accurate.

\section{Implicit solution algorithm}

Once in finite-volume and semidiscrete form, system (5) becomes:

$$
\Omega \frac{\partial Q}{\partial t}=-\mathbf{P} \operatorname{RES} \equiv-\mathbf{M M}_{\mathrm{m}}^{-1} \mathrm{RES}
$$

where RES represents the vector of residuals for the conservative variables and $\Omega$ the cell volume. Updating is done in terms of the viscous primitive variables 
HFF

12,6

644

$Q_{\mathrm{v}}$, namely pressure, temperature and the velocity components. This is done by multiplying the above system by $\mathbf{M}^{-1}$ to the left:

$$
\Omega \frac{\partial Q_{\mathrm{v}}}{\partial t}=-\mathbf{M}_{\mathrm{m}}^{-1} \text { RES }
$$

Systems (14) and (15) are equivalent. If an implicit numerical scheme is used to discretize the time derivative then:

$$
\Omega \frac{Q_{\mathrm{v}}^{\text {new }}-Q_{\mathrm{v}}^{\text {old }}}{\Delta t} \equiv \Omega \frac{\Delta Q_{\mathrm{v}}}{\Delta t}=-\mathbf{M}_{\mathrm{m}}^{-1} \operatorname{RES}^{\text {new }}
$$

After linearization about the old time level:

$$
\Omega \frac{\Delta Q_{\mathrm{v}}}{\Delta t}=-\mathbf{M}_{\mathrm{m}}^{-1}\left[\operatorname{RES}^{\text {old }}+\left(\frac{\partial \mathrm{RES}}{\partial Q_{\mathrm{v}}}\right)^{\text {old }} \Delta Q_{\mathrm{v}}\right]
$$

Re-arranging, multiplying by $\mathbf{M}_{\mathrm{m}}$ to the left, and dropping superscripts:

$$
\left(\mathbf{M}_{\mathrm{m}} \frac{\Omega}{\Delta t}+\frac{\partial \mathrm{RES}}{\partial Q_{\mathrm{v}}}\right) \Delta Q_{\mathrm{v}}=-\mathrm{RES}
$$

In equation (18) $\Delta Q_{\mathrm{v}}$ and RES are column vectors of dimension given by the total number $N$ of cells, where each element is an array of five components. The unsteady term matrix on the left-hand side is a block diagonal matrix (with $5 \times 5$ blocks). Jacobians of the residuals with respect to the variables are typically constructed by using a two-point stencil scheme at each interface. As a result, the second term within brackets is a sparse $N \times N$ matrix with nonzero elements only on seven diagonals, due to the grid structure.

Denoting the block element of the seven diagonals by $\mathbf{A}, \mathbf{B}_{\mathrm{I}}, \mathbf{B}_{\mathrm{J}}, \mathbf{B}_{\mathrm{K}}, \mathbf{C}_{\mathrm{I}}, \mathbf{C}_{\mathrm{J}}$ and $\mathrm{C}_{\mathrm{K}}$, then the solution algorithm can be written as:

$$
\begin{aligned}
& \left(\mathbf{M}_{\mathrm{m}} \frac{\Omega}{\Delta t}+\mathbf{A}\right) \Delta Q_{\mathrm{v}}(i, j, k)+\mathbf{B}_{\mathrm{I}} \Delta Q_{\mathrm{v}}(i-1, j, k)+\mathbf{B}_{\mathrm{J}} \Delta Q_{\mathrm{v}}(i, j-1, k) \\
& +\mathbf{B}_{K} \Delta Q_{\mathrm{v}}(i, j, k-1)+\mathbf{C}_{\mathrm{I}} \Delta Q_{\mathrm{v}}(i+1, j, k)+\mathbf{C}_{\mathrm{J}} \Delta Q_{\mathrm{v}}(i, j+1, k) \\
& +\mathbf{C}_{K} \Delta Q_{\mathrm{v}}(i, j, k+1)=-\operatorname{RES}(i, j, k)
\end{aligned}
$$

At each time step the linearization leads to the linear system (19), which is to be solved by an iterative method. When unconditional stability is achievable, equation (19) becomes a Newton method which is able to deliver quadratic convergence. It has to be noticed that, though not explicitly apparent from equation (18), preconditioning enters the linear system through the inviscid numerical flux (13), in addition to the unsteady term. People who make no use of characteristic-based schemes need little modifications of their codes. 


\section{Construction of Jacobian matrices}

The inviscid numerical flux at the interface between cells $(i, j, k)$ and $(i+1, j, k)$, given by equation (13), depends on the values of the primitive variables on both sides of the interface. With the convention that the cell surface normal $\overrightarrow{\Delta S}$ points towards the direction of increasing index $i$, the contribution $F_{i+1 / 2}^{*} \Delta S$ is to be added to the residual of the cell $(i, j, k)$, and subtracted to the residual of the cell $(i+1, j, k)$. Dropping subscripts $j$ and $k$ for the sake of clarity, and noting that the inviscid numerical flux depends only on the two states on the left and right of the cell interface, it follows:

$$
\begin{gathered}
\operatorname{RES}(i)=\operatorname{RES}(i)+\Delta S F_{i+1 / 2}^{*}\left(Q_{i}^{\mathrm{v}}, Q_{i+1}^{\mathrm{v}}\right) \\
\operatorname{RES}(i+1)=\operatorname{RES}(i+1)-\Delta S F_{i+1 / 2}^{*}\left(Q_{i}^{\mathrm{v}}, Q_{i+1}^{\mathrm{v}}\right)
\end{gathered}
$$

Differentiation of equation (13) with respect to $Q_{\mathrm{v}}$ allows the construction of the matrix block elements A, B's and C's, collecting all contributions by sweeping through all cell interfaces in the three structure directions:

$$
\begin{gathered}
\mathbf{A}_{i}=\mathbf{A}_{i}+\Delta S\left(H_{i}+D_{1+1 / 2}\right) / 2 \\
\mathbf{A}_{i+1}=\mathbf{A}_{i+1}-\Delta S\left(H_{i+1}-D_{1+1 / 2}\right) / 2 \\
\mathbf{B}_{i+1}=\mathbf{B}_{i+1}-\Delta S\left(H_{i}+D_{1+1 / 2}\right) / 2 \\
\mathbf{C}_{i}=\mathbf{C}_{i}+\Delta S\left(H_{i+1}-D_{1+1 / 2}\right) / 2
\end{gathered}
$$

where the Jacobian $H$, whose analytical expression is given by $\partial F / \partial Q_{\mathrm{v}}$, corresponds to the "central" part of the numerical flux expression. Differentiation with respect to $Q_{\mathrm{v}}$ of the second part of equation (13), representing the scheme matrix numerical dissipation, is based on the local linearization approach of Roe, where the contribution $D_{1+1 / 2}=\left[\mathbf{M}_{\mathrm{m}} \mathbf{R}_{p}^{\mathrm{v}}\left|\Lambda_{p}\right| \mathbf{L}_{p}^{\mathrm{v}}\right]$ is locally frozen, and evaluated at the cell interface based on the Roe's average state, a function of left $(i)$ and right $(i+1)$ states.

The viscous fluxes of system (1) are given by:

$$
\begin{gathered}
G_{\rho}=0 \\
G_{\rho u}=\tau_{x x} n_{x}+\tau_{x y} n_{y}+\tau_{x z} n_{z} \\
G_{\rho v}=\tau_{x y} n_{x}+\tau_{y y} n_{y}+\tau_{y z} n_{z} \\
G_{\rho w}=\tau_{x z} n_{x}+\tau_{y z} n_{y}+\tau_{z z} n_{z}
\end{gathered}
$$

Efficient parallel computations of fluid flows 
$\mathrm{HFF}$

12,6

646

$$
\begin{aligned}
G_{\rho E}= & \left(u \tau_{x x}+v \tau_{x y} n_{y}+w \tau_{x z} n_{z}+q_{x}\right) n_{x}+\left(u \tau_{x y}+v \tau_{y y} n_{y}+w \tau_{y z} n_{z}+q_{y}\right) n_{y} \\
& +\left(u \tau_{x z}+v \tau_{y z} n_{y}+w \tau_{z z} n_{z}+q_{z}\right) n_{z}
\end{aligned}
$$

Displaying the fluxes' dependence upon the primitive variables gives:

$$
\begin{gathered}
G_{\rho u}=\mu^{*}\left(2 \frac{n_{x}}{\partial x}+\frac{n_{y}}{\partial y}+\frac{n_{z}}{\partial z}\right) \partial u+\mu * \frac{n_{y}}{\partial x} \partial v+\mu * \frac{n_{z}}{\partial x} \partial w \\
G_{\rho v}=\mu *\left(\frac{n_{x}}{\partial x}+2 \frac{n_{y}}{\partial y}+\frac{n_{z}}{\partial z}\right) \partial v+\mu * \frac{n_{x}}{\partial y} \partial u+\mu * \frac{n_{z}}{\partial y} \partial w \\
G_{\rho w}=\mu^{*}\left(\frac{n_{x}}{\partial x}+\frac{n_{y}}{\partial y}+2 \frac{n_{z}}{\partial z}\right) \partial w+\mu^{*} \frac{n_{x}}{\partial z} \partial u+\mu^{*} \frac{n_{y}}{\partial z} \partial v \\
G_{\rho E}=\kappa^{*}\left(\frac{n_{x}}{\partial x}+\frac{n_{y}}{\partial y}+\frac{n_{z}}{\partial z}\right) \partial T+u^{*} G_{\rho u}+v^{*} G_{\rho v}+w^{*} G_{\rho w}
\end{gathered}
$$

where the superscript $*$ means frozen interface values. Differentiations of equation (23) with respect to $u, v, w$ and $T$ give the viscous fluxes contributions to the block Jacobians:

$$
\mathbf{A}_{i}=\mathbf{A}_{i}+\mathbf{G}
$$

$$
\begin{aligned}
\mathbf{A}_{i+1} & =\mathbf{A}_{i+1}+\mathbf{G} \\
\mathbf{B}_{i+1} & =\mathbf{B}_{i+1}-\mathbf{G} \\
\mathbf{C}_{i} & =\mathbf{C}_{i}-\mathbf{G}
\end{aligned}
$$

where the $5 \times 5$ matrix $\mathbf{G}$ is finally given by (all elements evaluated at cell interface):

$$
\mathbf{G}=\frac{\Delta S^{2}}{\Omega}\left(\begin{array}{ccccc}
0 & 0 & 0 & 0 & 0 \\
0 & \mu\left(1+n_{x}^{2}\right) & \mu n_{x} n_{y} & \mu n_{x} n_{z} & 0 \\
0 & \mu n_{x} n_{y} & \mu\left(1+n_{y}^{2}\right) & \mu n_{y} n_{z} & 0 \\
0 & \mu n_{x} n_{z} & \mu n_{y} n_{z} & \mu\left(1+n_{z}^{2}\right) & 0 \\
0 & \mu\left(u+V_{\mathrm{n}} n_{x}\right) & \mu\left(v+V_{\mathrm{n}} n_{y}\right) & \mu\left(w+V_{\mathrm{n}} n_{z}\right) & \kappa
\end{array}\right)
$$

where $\Omega$ respresents the cell average volume at the interface, and $\Delta S$ the 
interface area. It can be noted that the diagonal elements are positive definite. Near solid boundaries viscous contributions to Jacobians increase the stability of the solution algorithm.

\section{Parallel red-black relaxation scheme}

At each linearization step, equation (19) is solved with an iterative red-black relaxation scheme. The red-black scheme can be seen as the inherently parallel version of the Gauss-Seidel scheme: rather than choosing a sweep direction, first all red cells, then all black cells are treated in a chessboard pattern. The red loop reads as:

$$
\begin{aligned}
\Delta Q_{\mathrm{v}}^{n+1}(i, j, k)= & -\left(\mathbf{M}_{\mathrm{m}} \frac{\Omega}{\Delta t}+\mathbf{A}\right)^{-1}\left[\operatorname{RES}(i, j, k)+\mathbf{B}_{\mathrm{I}} \Delta Q_{\mathrm{v}}^{n}(i-1, j, k)\right. \\
& +\mathbf{B}_{\mathrm{J}} \Delta Q_{\mathrm{v}}^{n}(i, j-1, k)+\mathbf{B}_{K} \Delta Q_{\mathrm{v}}^{n}(i, j, k-1)+\mathbf{C}_{\mathrm{I}} \Delta Q_{\mathrm{v}}^{n}(i \\
& \left.+1, j, k)+\mathbf{C}_{\mathrm{J}} \Delta Q_{\mathrm{v}}^{n}(i, j+1, k)+\mathbf{C}_{K} \Delta Q_{\mathrm{v}}^{n}(i, j, k+1)\right]
\end{aligned}
$$

Before the red-cell loop, inversion of the block diagonal elements $\left(\mathbf{M}_{\mathrm{m}} \Omega / \Delta t+\right.$ A) is carried out for all blocks. In a multi-block code, the red loop must be made for all blocks in parallel (no matter whether in a single processor, or in a parallel machine). Then, for all blocks, the black-cell loop is given by:

$$
\begin{aligned}
\Delta Q_{\mathrm{v}}^{n+1}(i, j, k)= & -\left(\mathbf{M}_{\mathrm{m}} \frac{\Omega}{\Delta t}+\mathbf{A}\right)^{-1}\left[\operatorname{RES}(i, j, k)+\mathbf{B}_{\mathrm{I}} \Delta Q_{\mathrm{v}}^{n+1}(i-1, j, k)\right. \\
& +\mathbf{B}_{\mathrm{J}} \Delta Q_{\mathrm{v}}^{n+1}(i, j-1, k)+\mathbf{B}_{K} \Delta Q_{\mathrm{v}}^{n+1}(i, j, k-1) \\
& +\mathbf{C}_{\mathrm{I}} \Delta Q_{\mathrm{v}}^{n+1}(i+1, j, k)+\mathbf{C}_{\mathrm{J}} \Delta Q_{\mathrm{v}}^{n+1}(i, j+1, k) \\
& \left.+\mathbf{C}_{K} \Delta Q_{\mathrm{v}}^{n+1}(i, j, k+1)\right]
\end{aligned}
$$

where the contributions at the right-hand side are all at the new iteration level. At each iteration step $n$, in between the red and the black-cell loop, boundary updates $\left(\Delta Q_{\mathrm{v}} \mathrm{s}\right)$ are exchanged between adjacent blocks. The solution algorithm performance is totally independent of the number of blocks and can be easily ported to parallel machines, implementing MPI communications, with no loss of convergence rate.

\section{Turbulence model}

The Spalart \& Allmaras turbulence model (Spalart, 1994) is solved with an implicit algorithm decoupled from the main five equations. The solution algorithm is the same as the one just described. All Jacobian $5 \times 5$ block 
$\mathrm{HFF}$

12,6

648 matrices for $\mathbf{A}, \mathbf{B}$ and $\mathbf{C}$ become scalar values. Special care must be taken for the Jacobian contribution coming from the source and sink terms in the equation. The procedure described in detail in the cited reference is capable of assuring stability of the implicit time integration method.

\section{Results}

Four test cases covering a broad variety of applications are presented in the following. They include inviscid and turbulent test cases, incompressible hydrodynamics, liquid metal natural convection loops and supersonic nozzle gasdynamics. All cases were run with infinite CFL number starting from trivial uniform conditions. At each linearization step, the red-black relaxation scheme was iterated till convergence. This was achieved choosing a number of iteration steps about one and a half times the number of grid nodes normal to the solid walls. The resulting quasi-Newton method showed excellent efficiency and convergence history independent of the block mesh partition.

\subsection{Low speed turbulent flow over a backward facing step}

The work of Le et al. (1997) (DNS simulation) is used for comparison. The Reynolds number is 5,100 based on the step height $h$. The computational domain starts at $x h=10$ upstream of the step location, and ends at $x / h=20$ downstream. The resulting two-block mesh is made of $48 \times 40$ and $64 \times 80$ cells. A severe mesh stretching is provided close to the solid boundaries with a value of the non-dimensional grid spacing $y^{+}$of the order unity. The DNS simulation determines a re-attachment point at a distance of $x / h=6.28$ from the step, compared to $x h=5.75$ of the present Spalart \& Allmaras simulation.

Figure 1 shows the convergence history of streamwise momentum and turbulence model equations. Figure 2 shows velocity and Reynolds stress profiles at four different locations downstream of the step. The discrepancy at station $x h=6$ is affected by the earlier re-attachment of the present versus the DNS simulation.

\subsection{Buoyancy-driven annular loop}

A natural convection flow of a liquid metal (the eutectic Li17-Pb83 with $\mathrm{Pr}=$ $3.2 \times 10^{-2}$ ) is driven by a volumetric heat addition supplied in a sector of the domain at a rate $Q=8.4 \mathrm{~kW} / \mathrm{kg}$. The walls are kept at a constant temperature of $300 \mathrm{~K}$. The resulting Grashof number is $\mathrm{Gr}=2 \times 10^{6}$. The internal radius is $10^{-2} \mathrm{~m}$, while the external radius $2 \times 10^{-2} \mathrm{~m}$. The grid has 120 cells in the poloidal direction and 40 cells in the radial direction (Di Piazza, 2000; Di Piazza and Mulas, 2001).

Figure 3 shows the convence history. This is the only case where the simulation does not reach machine accuracy. As a matter of fact, an explicit run also showed the same stagnating convergence. Figure 4 shows the temperature field. The flow circulation is clockwise. The uniformly heated sector is visible in the figure. 


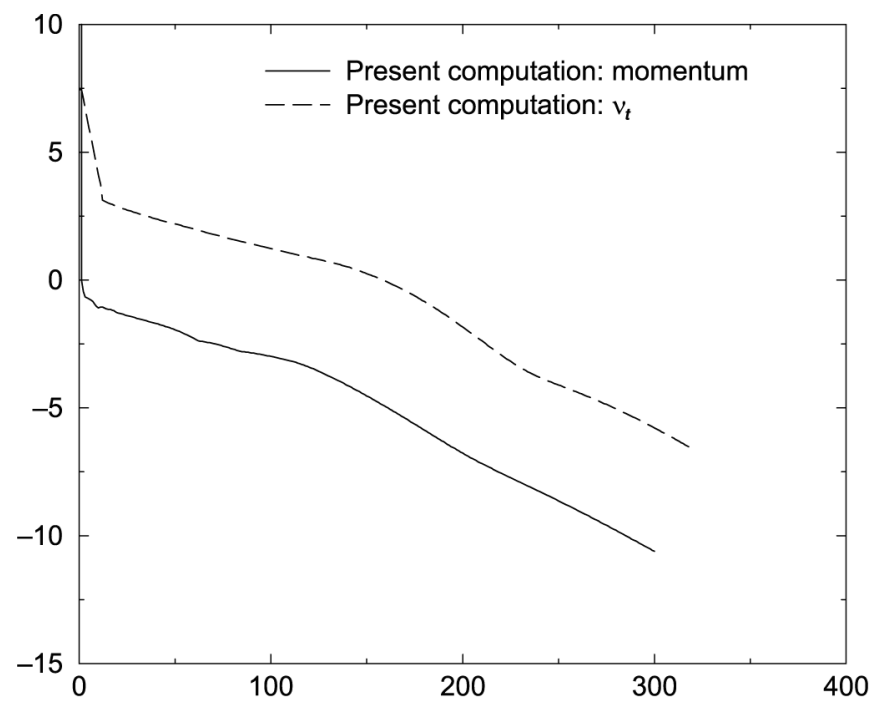

\section{Efficient parallel computations of fluid flows}

649

Figure 1.

Backward facing step: convergence

\subsection{Inviscid hydrodynamic flow over a ship hull}

A six-block $\mathrm{O}-\mathrm{H}$ type mesh is used to simulate the inviscid flow over a $\mathrm{S} 60$ type ship hull. The available towing tank measurements are provided by Longo (1996). The hull surface is described by 226 nodes in streamwise direction and 21 nodes in crosswise direction, with about a total of 150,000 cells.
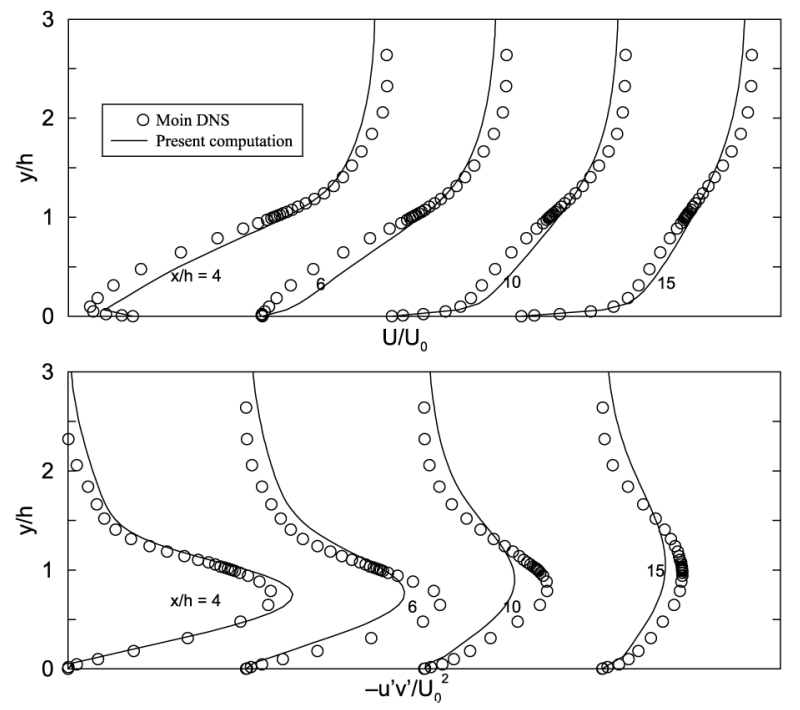

Figure 2. Backward facing step: velocity and Reynolds stress profiles 


\section{$\mathrm{HFF}$ \\ 12,6}

650

Figure 3.

Buoyancy driven loop: convergence

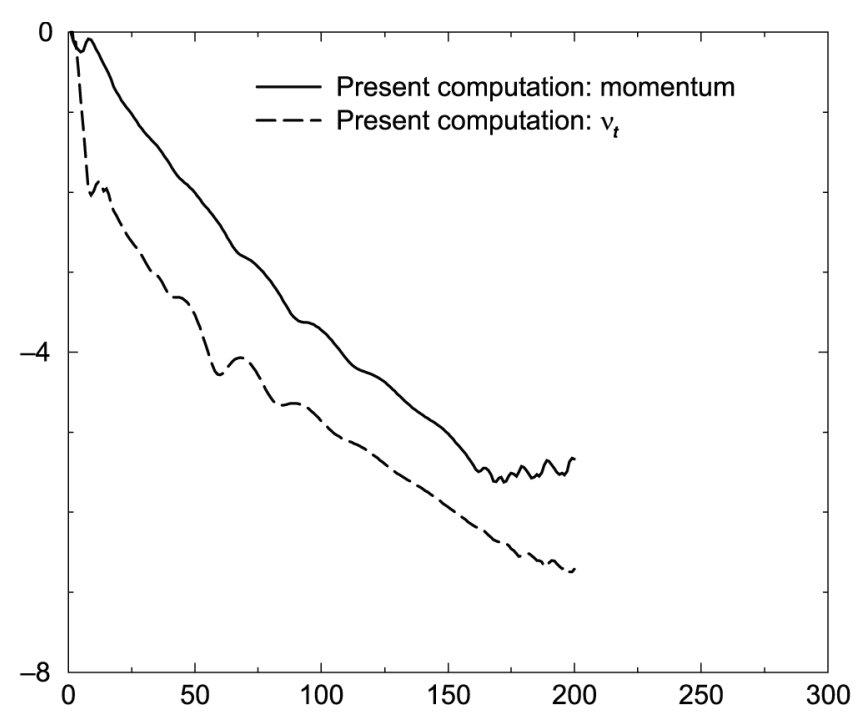

No effect of free surface is taken into account, so that measurement conditions for low Froude numbers (Mulas and Talice, 1999) are approached.

Figure 5 shows the convergence history. Figures 6 and 7 show the computed non dimensional lateral force and $z$-moment coefficients, compared to measurements for conditions with $\mathrm{Fr}=0.10$.

Figure 4.

Buoyancy driven loop: temperature field

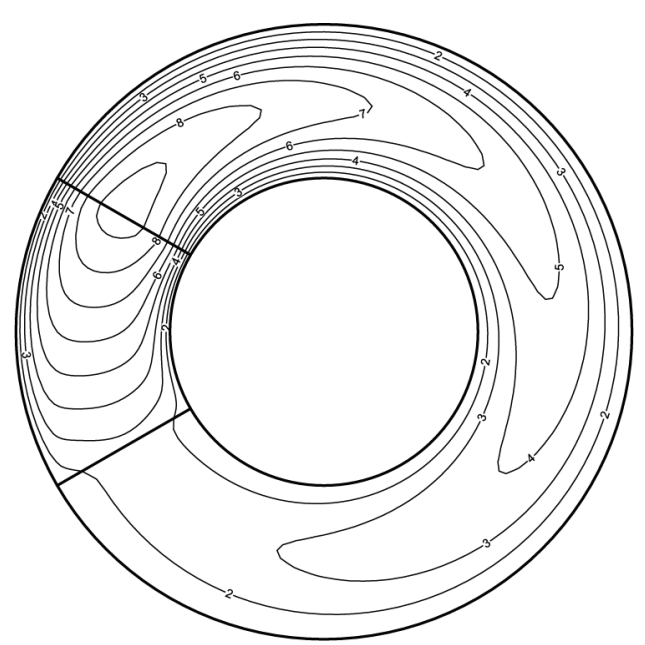




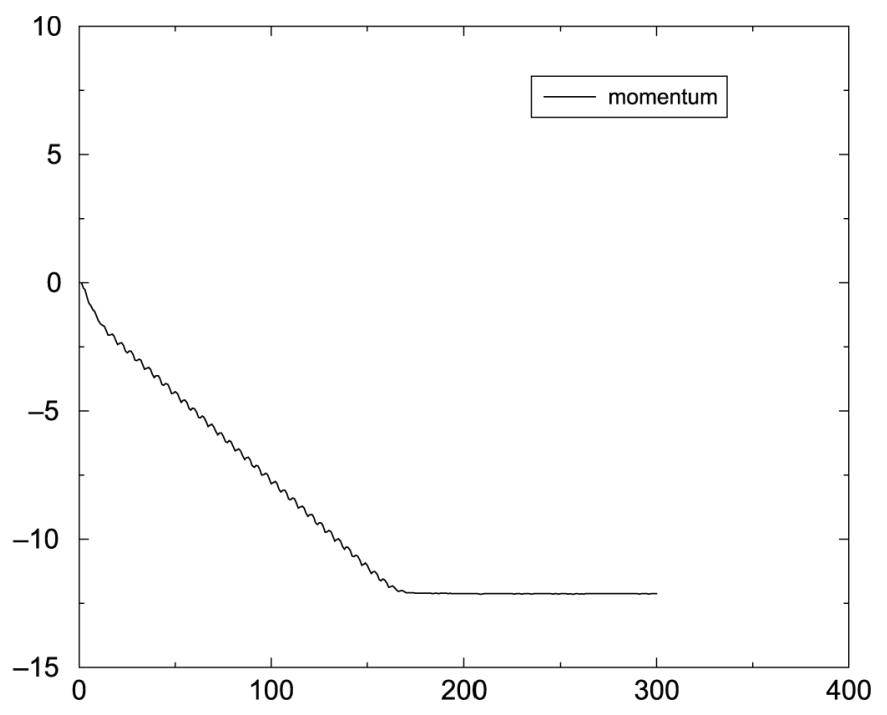

\section{Efficient parallel computations of fluid flows}

651

9.4 High enthalpy hydrogen rocket nozzle flow

The last case deals with a new concept of rocket propulsion, from an idea of Physics Nobel prize Carlo Rubbia (Augelli et al., 1999), capable of specific impulses of the order of $2000 \mathrm{~s}$. A hydrogen flow enters the stagnation chamber from a distributed inlet at about $1500 \mathrm{~K}$. As it flows through the chamber toward the convergent-divergent nozzle its temperature increases up to about $10,000 \mathrm{~K}$ due to direct energy conversion of the kinetic energy of the fission

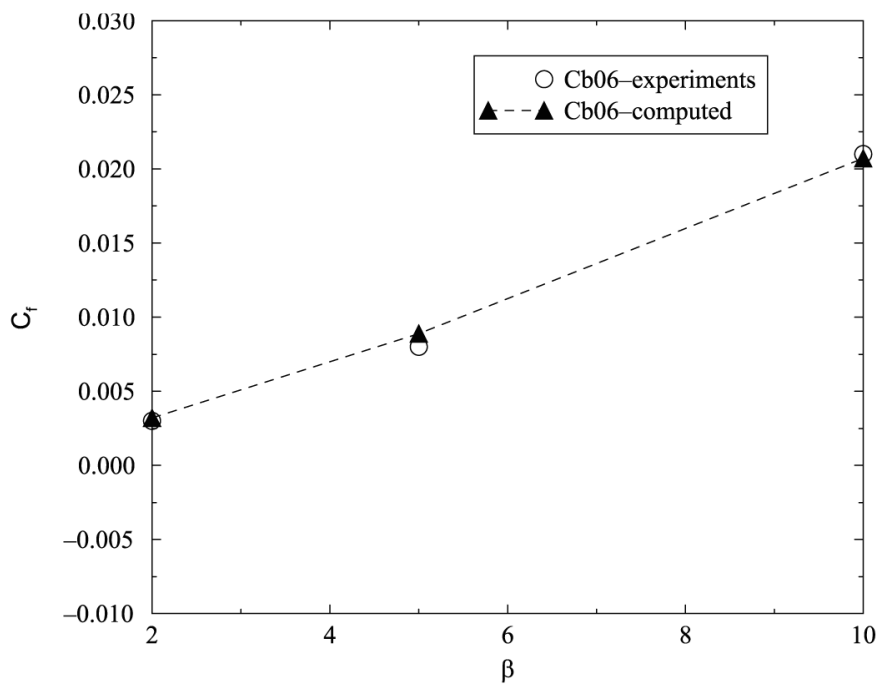

Figure 6.

Ship hull test case: non dimensional force coefficient vs drift angle 


\section{$\mathrm{HFF}$ \\ 12,6}

652

Figure 7.

Ship hull test case: non dimensional moment coefficient vs drift angle

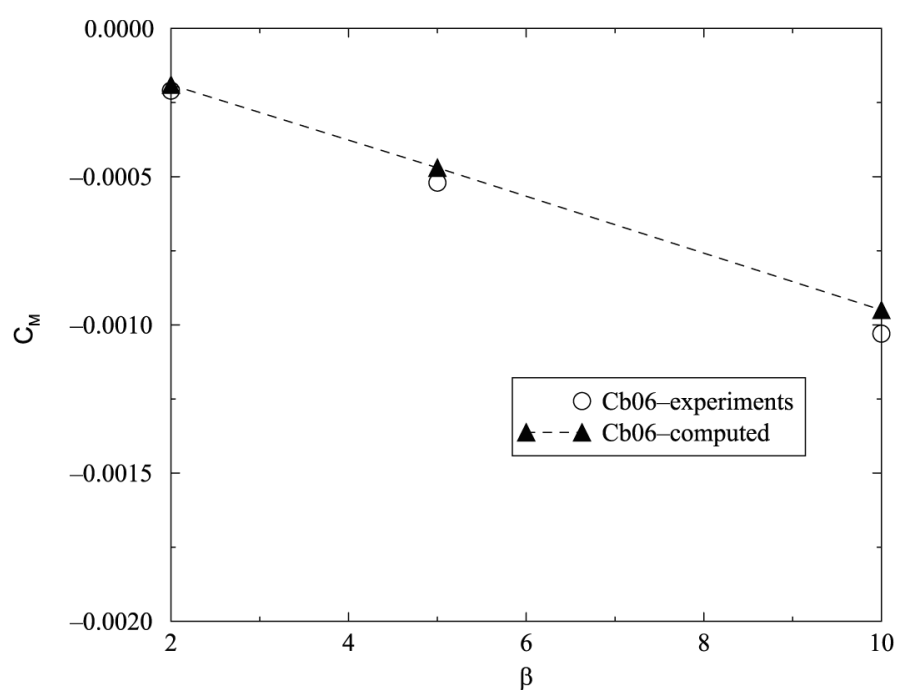

fragments originated from a thin layer of Americium 242 (smeared over the chamber walls and undergoing critical nuclear reaction) to hydrogen internal energy. This special case of volumetric heat addition was modelled with a Monte Carlo method coupled to the CFD code (Mulas, 2000; Leonardi et al., 2001).

This is an extremely stiff problem for three reasons: firstly because the whole spectrum of Mach number is present (inlet velocities are of the order of few centimeters per second, and exit velocities reach $20,000 \mathrm{~m} / \mathrm{s}$ ); then because the speed of sound of hydrogen at $10,000 \mathrm{~K}$ is about $10,000 \mathrm{~m} / \mathrm{s}$, with corresponding Mach number in the stagnation chamber of the order of $10^{-6}$; finally the transonic region at the nozzle throat represents another source of stiffness. Due to the operating temperature range, hydrogen equations of state are produced with a chemical equilibrium code in the form of look-up tables. As already mentioned, the output of the chemical equilibrium code takes into account the hydrogen dissociation and ionization processes occurring at temperature intervals depending on the pressure level. Radiative and absorption effects due to high temperature were also taken into account. The implicit preconditioned method, with infinite CFL number, allows an efficient flow calculation. Preconditioning automatically switches off as the flow locally reaches sonic conditions. Figure 8 shows the grey-scale temperature field and a few streamlines. Also shown, the domain block partition.

\section{Concluding remarks}

A unified numerical preconditioned method for the solution of fluid flow problems belonging to a wide class of applications of engineering interest has 


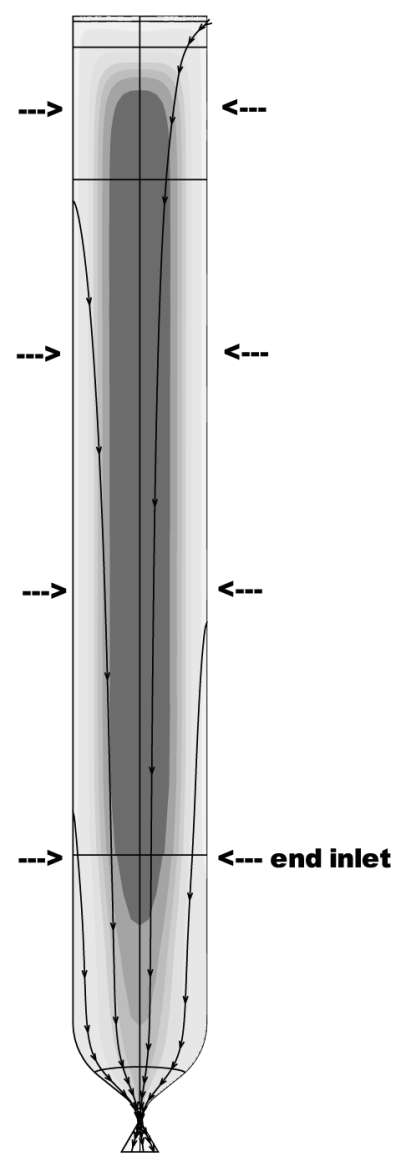

\section{Efficient parallel computations of fluid flows}

653

Figure 8.

Rocket nozzle: temperature field

been presented. The method is general for it allows a fluid definition by means of arbitrary equations of state and it is applicable to flows characterized by the whole range of Reynolds, Grashof and Mach numbers. The implicit discretization presented is able to stand infinite CFL numbers. The key feature for obtaining unconditional stability is the proper construction of the system matrix of the linearized time advancing scheme. The resulting linear system is solved at each linearization step with a red-black relaxation scheme. Pushing the linear system solution until convergence makes the scheme an efficient Newton method. Moreover, the red-black relaxation is intrinsically independent of the arbitrary multi-block partition of the flow domain and allows an easy multi-processor implementation with MPI technique. The second order TVD Roe's scheme keeps its outstanding accuracy features provided that the eigenvector decomposition is done in the preconditioned 
$\mathrm{HFF}$

12,6

654

world. General preconditioned eigenvector matrices have been developed for arbitrary thermodynamics. The numerical examples showed that the computational efficiency is independent of the class of fluid flow problem.

\section{References}

Augelli, M., Bignami, G.F., Bruno, C., Calligarich, E., De Maria, G., Mulas, M., Musso, C., Pellizzoni, A., Piperno, W., Piva, R., Procacci, B., Rosa-Clot, M. and Rubbia, C. (1999), "Report of the working group on a preliminary assessment of a new fission fragment heated propulsion concept and its applicability to manned missions to the planet Mars (Project 242)", ASI report, March 15, Roma.

Bijl, H. and Wesseling, P. (1996), "A method for the numerical solution of the almost incompressible Euler equations”, TU Delft Report 96-37, Delft

Bijl, H. and Wesseling, P. (1998), "A unified method for computing incompressible and compressible flows in boundary-fitted coordinates”, J. Comput. Phys., Vol. 141, pp. 153-73.

Bijl, H. and Wesseling, P. (2000), "Computation of unsteady flows at all speeds with a staggered scheme”, ECCOMAS 2000, 11-14 September, Barcelona.

Chorin, A.J. (1967), "A numerical methods for solving incompressible viscous flow problems", J. Comput. Phys., Vol. 2, pp. 12-26.

Choi, D. and Merkle, C.L. (1985), "Fully implicit solution of incompressible flows", AIAA J., Vol. 23, pp. 1518-24.

Choi, D. and Merkle, C.L. (1993), "The application of preconditioning to viscous flows", J. Comput. Phys., Vol. 105, pp. 207-23.

Di Piazza, I., "Prediction of free convection in liquid metals with internal heat generation and/or magnetohydrodynamic interactions," PhD thesis, University of Palermo, Italy.

Di Piazza, I. and Mulas, M. (2001), "Preconditiong tests on a buoyancy-driven anular loop using the CFD code Karalis", CRS4 TECH-REP 01/26.

Demirdøić, I., Lilek, Z. and Perić, M. (1993), “A collocated finite volume method for predicting flows at all speeds”, Int. J. Numer. Methods Fluids, Vol. 16, pp. 1029-50.

Guerra, J. and Gustafsson, B. (1986), "A numerical method for incompressible and compressible flow problems with smooth solutions”, J. Comput. Phys., Vol. 63, pp. 377-96.

Hirsch, Ch. (1990), Numerical Computation of Internal and External Flows, Wiley, New York Vol. 2.

Lee, D., "Local preconditioning of the Euler and Navier-Stokes equations," $\mathrm{PhD}$ thesis, University of Michigan.

Longo, J.F., "Effects of yaw model-scale ship flows," $\mathrm{PhD}$ thesis, University of Iowa.

Lee, D. and van Leer, B. (1993), "Progress in local preconditioning of the Euler and Navier-Stokes equations”, AIAA Paper 93-3328.

Le, H., Moin, P. and Kim, J. (1997), "Direct numerical simulation of turbulent flow over a backward facing step”, J. Fluid Mech., Vol. 330, pp. 349-74.

van Leer, B., Lee, W. and Roe, P. (1991), "Characteristic time-stepping or local preconditioning of the Euler equations”, AIAA Paper 91-1552.

Leonardi, E., Pili, P. and Varone, A. (2001), "Development of Montecarlo codes for the simulation of the trasport of Fission Fragments and the computation of radiative and absorption effects”, CRS4 TECH-REP 01/85.

Mulas, M. (2000), "Project 242: Fluid Dynamics final report”, CRS4 TECH-REP 00/94. 
Merkle, C.L. and Venkateswaran S. (1998), "The use of asymptotic expansions to enhance computational methods", AIAA Paper 98-2485.

Mulas, M. and Talice, M. (1999) Numerical determination of hydrodynamic coefficients for manoeuvring without free surface effects, CRS4-TECH-REP 99/19, 1999.

Merkle, C.L., Sullivan, J.Y., Buelow, P.E.O. and Venkateswaran, S. (1998), “Computation of flows with arbitrary equation of state”, AIAA J., Vol. 36 No. 4, pp. 515-21.

Peyret, R. and Viviand, H. (1985), "Pseudo unsteday methods for inviscid or viscous flow compuatations", in Casci, C., (Eds), Recent Advances in the Aerospace Sciences, Plenum, New York, pp. 41-71.

Patnaik, G., Guirguis, R.H., Boris, J.P. and Oran, E.S. (1987), “A barely implicit correction for fluxcorrected transport”, J. Comput. Phys., Vol. 71 No. 1, pp. 1-20.

Roe, P.L. (1981), “Approximate riemann solvers, parameters vectors and difference schemes”, J. Comput. Phys., Vol. 43, pp. 357-72.

Spalart, P.R. and Allmaras, S.R. (1992), "A one-equation turbulence model for aerodynamics flows" AIAA Paper 92-0439.

Turkel, E. (1984), “Acceleration to a steady state for the Euler equations”, ICASE Rep., pp. 84-32.

Turkel, E. (1987), "Preconditioned methods for solving the incompressible and low speed compressible equations", J. Comput. Phys., Vol. 72, pp. 277-98.

Venkateswaran, S., Weiss, S. and Merkle, C.L. (1992), "Propulsion related flowfields using the preconditioned Navier-Stokes equations”, AIAA Paper 92-3437.

Weiss, J.M. and Smith, W.A. (1995), "Preconditioning applied to variable and constant density flow”, AIAA J., Vol. 33 No. 11, pp. 2050-7.

Weiss, J.M., Maruszewski, J.P. and Smith, W.A. (1997), "Implicit solution of the Navier-Stokes equations on unstructured meshes", AIAA Paper 97-2103.

Wesseling, P., van der Heul, D.R. and Vuik, C. (2000), "Unified methods for computing compressible and incompressible flows”, ECCOMAS 2000, 11-14 September, Barcelona.

\section{Appendix. Eigenvectors and eigenvalues}

The Jacobian matrices of primitive variables with respect to conservative variables and vice versa are given by:

$$
\mathbf{M}=\frac{\partial Q}{\partial Q_{\mathrm{v}}}=\left(\begin{array}{ccccc}
\rho_{p} & 0 & 0 & 0 & \rho_{T} \\
u \rho_{p} & \rho & 0 & 0 & u \rho_{T} \\
v \rho_{p} & 0 & \rho & 0 & v \rho_{T} \\
w \rho_{p} & 0 & 0 & \rho & w \rho_{T} \\
\rho_{p}-\left(1-\rho h_{p}\right) & \rho u & \rho v & \rho w & H \rho_{T}+\rho h_{T}
\end{array}\right)
$$

\section{Efficient parallel computations of fluid flows}


$\mathrm{HFF}$

12,6

656

$$
\mathbf{M}^{-1}=\frac{\partial Q_{\mathrm{v}}}{\partial Q}=\left(\begin{array}{ccccc}
\frac{\rho h_{T}+\rho_{T}\left(H-V^{2}\right)}{d} & \frac{\rho_{T}}{d} u & \frac{\rho_{T}}{d} v & \frac{\rho_{T}}{d} w & -\frac{\rho_{T}}{d} \\
-\frac{u}{\rho} & \frac{1}{\rho} & 0 & 0 & 0 \\
-\frac{v}{\rho} & 0 & \frac{1}{\rho} & 0 & 0 \\
-\frac{w}{\rho} & 0 & 0 & \frac{1}{\rho} & 0 \\
\frac{-\rho_{p}\left(H-V^{2}\right)+1-\rho h_{p}}{d} & -\frac{\rho_{p}}{d} u & -\frac{\rho_{p}}{d} v & -\frac{\rho_{p}}{d} w & \frac{\rho_{p}}{d}
\end{array}\right)
$$

where $d$ is given by $d=\rho \rho_{p} h_{T}+\rho_{T}\left(1-\rho h_{p}\right)$. The modified versions $\mathbf{M}_{\mathrm{m}}$ and $\mathbf{M}_{\mathrm{m}}^{-1}$ are obtained from non modified ones (equations (28) and (29)) substituting $\rho_{p}$ with $\rho_{p}^{\mathrm{m}}$, so that $d$ becomes $d^{\mathrm{m}}$ accordingly.

The system eigenvalues, elements of $\Lambda_{p}$ of equation (13) are $\lambda_{1,2,3}=V_{\mathrm{n}} \equiv u n_{x}+v n_{y}+w n_{z}$ and:

$$
\lambda_{4,5}=V_{\mathrm{n}}\left(\frac{d+d^{\mathrm{m}}}{2 d^{\mathrm{m}}}\right) \pm \sqrt{V_{n}^{2}\left(\frac{d-d^{\mathrm{m}}}{2 d^{\mathrm{m}}}\right)^{2}+\frac{\rho h_{T}}{d^{\mathrm{m}}}} .
$$

Non-preconditioned eigenvalues are recovered when $d=d^{\mathrm{m}}$ noticing that the speed of sound is given by $c=\sqrt{\rho h_{T} / d}$. The matrix $\mathbf{L}_{p}^{v}$ of left eigenvectors is given by:

$$
\mathbf{L}_{p}^{\mathrm{v}}=\left(\begin{array}{ccccc}
-\frac{\rho_{T}}{d^{\mathrm{m}}} n_{x} & 0 & \frac{\mathbf{A}}{\Delta^{\mathrm{m}}} n_{z} & -\frac{\mathbf{A}}{\Delta^{\mathrm{m}}} n_{y} & -\frac{\mathbf{A}}{T} n_{x} \\
-\frac{\rho_{T}}{d^{\mathrm{m}}} n_{y} & -\frac{\mathbf{A}}{\Delta^{\mathrm{m}}} n_{z} & 0 & \frac{\mathbf{A}}{\Delta^{\mathrm{m}}} n_{x} & -\frac{\mathbf{A}}{T} n_{y} \\
-\frac{\rho_{T}}{d^{\mathrm{m}}} n_{z} & \frac{\mathbf{A}}{\Delta^{\mathrm{m}}} n_{y} & -\frac{\mathbf{A}}{\Delta^{\mathrm{m}}} n_{x} & 0 & -\frac{\mathbf{A}}{T} n_{z} \\
\frac{1}{2}+\mathbf{B} & \frac{1}{2} \frac{\mathbf{A}}{\Delta^{\mathrm{m}}} n_{x} & \frac{1}{2} \frac{\mathbf{A}}{\Delta^{\mathrm{m}}} n_{y} & \frac{1}{2} \frac{\mathbf{A}}{\Delta^{\mathrm{m}}} n_{z} & 0 \\
\frac{1}{2}-\mathbf{B} & -\frac{1}{2} \frac{\mathbf{A}}{\Delta^{\mathrm{m}}} n_{x} & -\frac{1}{2} \frac{\mathbf{A}}{\Delta^{\mathrm{m}}} n_{y} & -\frac{1}{2} \frac{\mathbf{A}}{\Delta^{\mathrm{m}}} n_{z} & 0
\end{array}\right)
$$

where:

$$
\Delta^{\mathrm{m}}=\sqrt{V_{n}^{2}\left(\frac{d-d^{\mathrm{m}}}{2 d^{\mathrm{m}}}\right)^{2}+\frac{\rho h_{T}}{d^{\mathrm{m}}}} ; \quad \mathbf{A}=\frac{\rho^{2} h_{T}}{d^{\mathrm{m}}} ; \quad \mathbf{B}=\frac{1}{4} \frac{V_{\mathrm{n}}}{\Delta^{\mathrm{m}}} \frac{d-d^{\mathrm{m}}}{d^{\mathrm{m}}}
$$

In case of no preconditioning:

$$
d^{\mathrm{m}}=d \Rightarrow \Delta^{\mathrm{m}}=\Delta=c \quad \mathbf{A}=\rho c^{2} \quad \text { and } \quad \mathbf{B}=0
$$

It has to be noticed that, when deriving the eigenvector matrices, the first three elements of the fifth column of $\mathbf{L}_{p}^{\mathrm{v}}$ take the following form: 


$$
\frac{\rho \rho_{T} h_{T}}{d^{\mathrm{m}}\left(1-\rho h_{p}\right)}
$$

which would generate a $0 / 0$ term when using incompressible fluids with $\beta=\xi=0$. However, for any pure substance, the above term is equivalent to $\rho^{2} h_{T} / d^{\mathrm{m}} T$.

In the matrix $\mathbf{L}_{b}^{v}$, the first three rows represent linear combinations of the entropy and the two shear waves, all of them propagating with characteristic speed given by $\lambda_{1,2,3}=V_{\mathrm{n}}$. The last two rows represent the two acoustic waves with caracteristic speeds given by $\lambda_{4}$ and $\lambda_{5}$. When the ideal gas law is considered (with no preconditioning), the well known left eigenvector matrix $\mathbf{L}^{v}$ in primitive variables is recovered

$$
\mathbf{L}^{v}=\left(\begin{array}{ccccc}
(\gamma-1) n_{x} & 0 & \rho c n_{z} & -\rho c n_{y} & -\frac{\rho c^{2}}{T} n_{x} \\
(\gamma-1) n_{y} & -\rho c n_{z} & 0 & \rho c n_{x} & -\frac{\rho c^{2}}{T} n_{y} \\
(\gamma-1) n_{z} & \rho c n_{y} & -\rho c n_{x} & 0 & -\frac{\rho c^{2}}{T} n_{z} \\
\frac{1}{2} & \frac{1}{2} \rho c n_{x} & \frac{1}{2} \rho c n_{y} & \frac{1}{2} \rho c n_{z} & 0 \\
\frac{1}{2} & -\frac{1}{2} \rho c n_{x} & -\frac{1}{2} \rho c n_{y} & -\frac{1}{2} \rho c n_{z} & 0
\end{array}\right)
$$

The matrix $\mathbf{M}_{\mathrm{m}} \mathbf{R}_{p}^{v}$ is given by equation (36): without preconditioning and when the ideal gas law is applied, it reduces to $\mathbf{R}$, the Euler right eigenvector matrix in conservative variables:

$\mathbf{R}=\frac{1}{c^{2}}\left(\begin{array}{ccccc}n_{x} & n_{y} & n_{z} & 1 & 1 \\ u n_{x} & u n_{y}-c n_{z} & u n_{z}+c n_{y} & u+c n_{x} & u-c n_{x} \\ v n_{x}+c n_{z} & v n_{y} & v n_{z}-c n_{x} & v+c n_{y} & v-c n_{y} \\ w n_{x}-c n_{y} & w n_{y}+c n_{x} & w n_{z} & w+c n_{z} & w-c n_{z} \\ \frac{V^{2}}{2} n_{x}+c\left(v n_{z}-w n_{y}\right) & \frac{V^{2}}{2} n_{y}+c\left(w n_{x}-u n_{z}\right) & \frac{V^{2}}{2} n_{z}+c\left(u n_{y}-v n_{x}\right) & H+c V_{\mathrm{n}} & H-c V_{\mathrm{n}}\end{array}\right)$

$$
\mathbf{M}_{\mathrm{m}} \mathbf{R}_{p}^{v}=\frac{d^{\mathrm{m}}}{\rho h_{T}}\left(\begin{array}{ccccc}
-\frac{T \rho_{T}}{\rho} n_{x} & -\frac{T \rho_{T}}{\rho} n_{y} & -\frac{T \rho_{T}}{\rho} n_{z} & 1 \\
-\frac{T \rho_{T}}{\rho} u n_{x} & -\frac{T \rho_{T}}{\rho} u n_{y}-\Delta^{\mathrm{m}} S_{z} & -\frac{T \rho_{T}}{\rho} u n_{z}+\Delta^{\mathrm{m}} S_{y} & u+\left(\mathbf{F}+\Delta^{\mathrm{m}}\right) n_{x} & u+\left(\mathbf{F}-\Delta^{\mathrm{m}}\right) n_{x} \\
-\frac{T \rho_{T}}{\rho} v n_{x}+\Delta^{\mathrm{m}} S_{z} & -\frac{T \rho_{T}}{\rho} v n_{y} & -\frac{T \rho_{T}}{\rho} v n_{z}-\Delta^{\mathrm{m}} S_{x} & v+\left(\mathbf{F}+\Delta^{\mathrm{m}}\right) n_{y} & v+\left(\mathbf{F}-\Delta^{\mathrm{m}}\right) n_{y} \\
-\frac{T \rho_{T}}{\rho} w n_{x}-\Delta^{\mathrm{m}} S_{y} & -\frac{T \rho_{T}}{\rho} w n_{y}+\Delta^{\mathrm{m}} S_{x} & -\frac{T \rho_{T}}{\rho} w n_{z} & w+\left(\mathbf{F}+\Delta^{\mathrm{m}}\right) n_{z} & w+\left(\mathbf{F}-\Delta^{\mathrm{m}}\right) n_{z} \\
-T\left(\frac{H \rho_{T}}{\rho}+h_{T}\right) n_{x}+\Delta^{\mathrm{m}}\left(v n_{z}-w n_{y}\right) & -T\left(\frac{H \rho_{T}}{\rho}+h_{T}\right) n_{y}+\Delta^{\mathrm{m}}\left(w n_{x}-u n_{z}\right) & -T\left(\frac{H \rho_{T}}{\rho}+h_{T}\right) n_{z}+\Delta^{\mathrm{m}}\left(u n_{y}-v n_{x}\right) & H+\left(\mathbf{F}+\Delta^{\mathrm{m}}\right) V_{\mathrm{n}} & H+\left(\mathbf{F}-\Delta^{\mathrm{m}}\right) V_{\mathrm{n}}
\end{array}\right)
$$

where $\mathbf{F}=-\frac{1}{2} V_{\mathrm{n}}\left(d-d^{\mathrm{m}} / d^{\mathrm{m}}\right)$. 